\title{
Nomen omen. Un museo a Predappio?
}

Le molte polemiche sollevate dal progetto del museo storico a Predappio sono la dimostrazione - l'ennesima - della difficoltà di metabolizzare l'esperienza del fascismo, di fare i conti fino in fondo con una pagina cruciale della storia italiana: una vicenda che sembra ancora pesare non poco sull'identità e la coscienza civile del paese, nonostante il tempo ormai considerevole che ci separa dal Ventennio. Leggendo i tanti interventi che si sono susseguiti nella prima fase della polemica, l'impressione che si ricava è che i riflettori siano stati puntati molto sugli aspetti pubblici dell'operazione, meno sul contenitore in sé. Nulla di strano, considerando le tante implicazioni collegate a un luogo - Predappio-così fortemente connotato nell'immaginario collettivo. Ne è derivata peraltro un'attenzione forse troppo modesta alle specificità insite nella narrazione museale della storia, che oggi è chiamata a confrontarsi con i modelli ereditati dalle esperienze del passato e con le nuove modalità della comunicazione e trasmissione della storia.

È noto che i musei storici si reggono su un dispositivo narrativo che li colloca all'incrocio di numerose istanze. Essi svolgono una funzione scientifica rilevante, specialmente quando la loro istituzione si collega a (o scaturisce da) esigenze di conservazione e valorizzazione di collezioni documentarie specifiche. D'altro lato, non meno importanti sono le valenze didattiche e quelle latamente educative, che proiettano i musei al centro delle battaglie culturali sul passato di ogni nazione. Il punto di equilibrio tra queste due dimensioni non è mai scontato, risentendo dei condizionamenti prodotti dai diversi contesti politici e culturali. 
Nel corso dell'Ottocento, i musei storici hanno ampiamente contribuito alla costruzione delle identità nazionali, integrandosi con i tanti altri canali di una politica della memoria impregnata di istanze pedagogico-patriottiche. In quella fase la spinta educativa è stata perciò particolarmente intensa. In Italia, i musei del Risorgimento, la cui diffusione si registra a partire dagli anni Ottanta del XIX secolo, sono la tipologia che meglio di altre restituisce la mescolanza di obiettivi e gli interessi molteplici che gravavano sulle attività museali. Non è un caso che le principali tappe della storia nazionale (colonialismo, Grande guerra, fascismo, Resistenza) siano state a lungo incorporate nelle sale dei musei del Risorgimento, secondo una logica continuista che tendeva, con obiettivi diversi a seconda delle stagioni, a legittimare il presente nel suo legame con il mito di fondazione dello stato unitario.

Negli ultimi decenni, le politiche museali, le teorie e le pratiche degli spazi espositivi sono evidentemente mutate, respirando positivamente le nuove sensibilità storiografiche. Resta comunque centrale, una volta dato per scontato il necessario rigore scientifico, la creazione di un "effetto coinvolgimento" che sia capace di investire anche la sfera emotiva, sollecitando l'intensità della partecipazione e con essa la fruizione del messaggio culturale.

Il nesso comporta problemi di non facile soluzione. Sul versante propriamente tecnico-operativo si sono moltiplicati gli esempi di allestimenti che sfruttano le innovazioni tecnologiche e puntano a una interazione diretta con i visitatori tramite le risorse multimediali. Gli studenti continuano a essere un destinatario privilegiato dell'azione educativa del museo, che ne incoraggia il coinvolgimento affidandosi a una narrazione imperniata sull'attivazione simultanea dei sensi, con particolare riferimento alle sfere visiva, tattile, uditiva. Il Museo del Novecento (M9), che sarà inaugurato a Mestre nel dicembre 2018, dovrebbe costituire un modello aggiornato alle filosofie espositive più moderne.

In taluni casi, possono sorgere complicazioni di natura più propriamente politica e ideologica. Il museo a Predappio rientra in questa casistica, per i motivi facilmente intuibili connessi all'accesa discussione sul passato dell'Italia unita, che ha assunto toni e caratteri nuovi a partire dalla crisi della "repubblica dei partiti". Va notato, per inciso, che in questa revisione del rapporto con la propria storia l'Italia sembra mostrare sempre di più singolari analogie con la situazione dei paesi dell'Europa centro-orientale all'indomani della guerra fredda: qui il fallimento dall'esperienza comunista e la legittimazione dei nuovi stati e governi sono stati accompagnati da un fortissimo investimento in termini di politiche e narrazioni memoriali, che hanno spesso rovesciato le letture precedenti, conservandone l'impianto assertivo e il tono poco permeabile alla complessità dei con- 
testi. Benché componente dell'alleanza atlantica uscita vincitrice dello scontro bipolare, l'Italia ne è uscita in una condizione politica e psicologica più simile a quella degli stati sconfitti (in una versione che richiama il post-1918); come questi ultimi si è trovata a gestire un mutamento epocale che, insieme al declino dei partiti storici, ha investito narrazioni consolidate, imperniate sulla centralità del Risorgimento e della Resistenza nel discorso pubblico.

L'incertezza politica e il disorientamento culturale propri di questa lunga fase di transizione non sembrano irrilevanti a fronte delle molte perplessità che il museo a Predappio ha sollevato tra gli addetti ai lavori. Pur riconoscendo le buone intenzioni dei promotori, le voci critiche temono che il museo possa contribuire a relativizzare il giudizio storico su Mussolini e sul fascismo e a ridurre la forza nel discorso pubblico di un sentimento antifascista già molto indebolito dagli attacchi ricevuti in questi ultimi decenni.

Le preoccupazioni non sono infondate, alla luce della confusione che accompagna l'evoluzione del concetto e della pratica della democrazia e dei rigurgiti di intolleranza che permeano la società italiana. Si tratta peraltro di capire se le legittime considerazioni di natura etico-politica siano in sé sufficienti a ostacolare un progetto che intenda fondare su robuste fondamenta storiografiche lo stesso ruolo civico del museo: laddove quest'ultimo va inteso anche come una «metafora sociale, cioè un mezzo attraverso il quale la società rappresenta il suo rapporto con la propria storia e con quella di altre culture» (dalla relazione allegata al progetto museografico).

Gli studi sul fascismo hanno raggiunto negli ultimi decenni risultati ragguardevoli. Permangono fisiologiche differenze di interpretazioni su alcune questioni generali e particolari, ma nessun studioso potrebbe oggi tentare di accreditarsi riproponendo letture moralistiche o viziate da preconcetti politici e ideologici. Il progetto scientifico che accompagna il museo a Predappio, sintetizzando la natura compiutamente storica del fenomeno fascista, rispecchia lo stato di avanzamento degli studi e si propone di tradurli nella presentazione museale dei passaggi più rilevanti della società italiana durante il ventennio. Qualcuno ha fatto notare alcune assenze rilevanti (ad esempio, le vicende del confine dell'alto Adriatico, con le relative politiche di snazionalizzazione e l'antislavismo; il corporativismo come "terza via" fascista rispetto alle aporie del capitalismo e del comunismo). È auspicabile che possano essere recuperate in sede di revisione dell'allestimento. Ma nel complesso, è evidente che a Predappio non si vuole istituire un "museo del fascismo", come frettolosamente si è detto agli esordi della polemica, quando, stante ancora l'incertezza sulle coordinate del progetto, la discussione fu da subito incanalata su piani diversi da quelli propriamente scientifico-culturali. Né pare 
convincente il timore che un museo siffatto possa rilanciare una lettura personalistica del regime, in termini di "mussolinismo" più che di fascismo. Ora sappiamo che dentro gli spazi dell'ex Casa del fascio di Predappio si svilupperà un itinerario che intende fotografare l'Italia nella transizione tra guerra e dopoguerra, illustrarne i processi di trasformazione sotto la spinta dell'ambizione totalitaria del regime, registrarne risultati e limiti, fino al fallimentare e tragico esito negli anni della Seconda guerra mondiale.

Detto ciò, a nessuno sfugge che il nodo della questione sia Predappio. Nel luogo, e in ciò che esso rappresenta, si annida il vero spauracchio. Il paese - autentico nomen omen-assorbe e incorpora per metonimia i fantasmi del passato e quelli nuovi del presente, proiettando sul futuro l'ombra di Mussolini e di quanti continuano a venerarne la memoria $\mathrm{o}$, più velatamente, a proporre una visione bonaria ed edulcorata del ventennio. L'opposizione al museo si è dunque concentrata su ciò che il paese natale del duce ha rappresentato nel corso del ventennio e poi nei decenni dell'Italia repubblicana. Un piccolo borgo, catapultato agli onori della cronaca negli anni Venti-Trenta, fu celebrato come la Betlemme della nuova religione politica, meta di pellegrinaggi di dopolavoristi, studenti, militari, semplici cittadini dell'Italia in camicia nera. Dopo il 1945 le falangi si sono drasticamente assottigliate: il viaggio a Predappio è diventato un appuntamento obbligatorio per i militanti dell'estrema destra, alla ricerca di luoghi, liturgie, simboli e memorie su cui agganciare il loro senso di appartenenza ideologica. D'altra parte, a partire dal 1957, quando la salma di Mussolini è tornata nella cripta di famiglia dopo un lungo e tormentato viaggio-narrato con efficacia da Sergio Luzzatto -, i rituali funerari qui inscenati hanno comunque raggiunto dimensioni non trascurabili, che non accennano a diminuire.

Sono obiezioni che, anche in questo caso, vanno considerate con attenzione, perché nessuno può ignorare cosa abbia significato Predappio nel discorso pubblico nazionale e ciò che ancora oggi il suo nome evochi nella percezione degli italiani. Occorre piuttosto capire se un museo storico possa contribuire a modificare questo "destino", e se abbia un senso storico e culturale rendere Predappio un luogo che non sia associato automaticamente al culto di Mussolini e alle parate rituali dei suoi nostalgici. Nel caso del museo, la scelta di fare di Predappio un luogo di conoscenza critica si nutre evidentemente di una strategia "aggressiva", che punta a colpire la mitologia alle fondamenta, nel cuore stesso degli spazi urbanistico-architettonici voluti dal regime, e ovviamente con il supporto di necessari percorsi didattici, affidati a persone competenti e specializzate in tema di storia d'Italia e di racconto museale. 
È una scommessa tutt'altro che agevole, che andrà monitorata in itinere e messa costantemente alla prova dei risultati conseguiti nella sua interazione con la società. A un secolo di distanza dalla fondazione dei Fasci di combattimento, mi pare tuttavia un'operazione che non meriti di essere stigmatizzata con argomentazioni che rischiano di riportare la discussione sul fascismo a una lettura interamente schiacciata sulla sua natura violenta e liberticida. Quest'ultima è innegabile, ha avuto un ruolo decisivo e configura un dato acquisito in campo storiografico. Isolarne il significato varrebbe però a smarrire l'enorme mole di ricerca accumulata per evidenziare il connubio di violenza, modernizzazione, mobilitazione sociale che marca la novità della dittatura totalitaria e la cui comprensione storica non può essere ignorata nello stesso percorso di formazione di una consapevole cittadinanza democratica. I tanti, inquietanti fenomeni di intolleranza, violenza e razzismo che l'attualità restituisce non dovrebbero essere un freno alla costruzione di categorie analitiche sempre più raffinate. La capacità di un paese di affrontare criticamente il proprio passato si misura anche dalla disponibilità (e dal coraggio) di nutrire di comprensione storica le tensioni etico-politiche del presente, più che dall'adozione di scorciatoie basate sulla condanna morale e politica: la quale, se può soddisfare nell'immediato il nostro bisogno di arginare derive e pulsioni antidemocratiche, contribuisce a sedimentare la rimozione di pagine cruciali, anche quando oscure, di una storia che è di tutti.

Se un museo storico a Predappio saprà muoversi in questa direzione, con i linguaggi e le competenze necessari a sostenere un'opera ambiziosa e rischiosa, il suo ruolo potrebbe rivelarsi prezioso sul piano della narrazione storica non meno che su quello dello sviluppo di una cultura democratica fondata sulla conoscenza critica del nostro passato. 